\title{
Continuous subcutaneous insulin infusion reduces neonatal risk in pregnant women with type 1 diabetes mellitus
}

\author{
Urszula Mantaj ${ }^{1}$, Pawel Gutaj ${ }^{1}$, Katarzyna Ozegowska ${ }^{2}$, Agnieszka Zawiejska ${ }^{1}$, \\ Katarzyna Wroblewska-Seniuk ${ }^{3}$, Danuta Olejniczak ${ }^{4}$, Ewa Wender-Ozegowska ${ }^{1}$ \\ ${ }^{1}$ Department of Reproduction, Poznan University of Medical Sciences, Poznan, Poland \\ ${ }^{2}$ Department of Infertility and Reproductive Endocrinology, Poznan University of Medical Sciences, Poznan, Poland \\ ${ }^{3}$ Department of Newborns'Infectious Diseases, Poznan University of Medical Sciences, Poznan, Poland \\ ${ }^{4}$ Gynecological and Obstetrics Hospital of the Poznan University of Medical Sciences, Poznan, Poland
}

\begin{abstract}
Objectives: An attempt was made to demonstrate the superiority of the treatment model using continuous subcutaneous insulin infusion (CSII) over multiple daily injections (MDI) of insulin in achieving a successful pregnancy outcome and good newborn's condition in patients with type 1 diabetes.

Material and methods: The study included 297 infants born to type 1 diabetic patients; 175 patients were treated with MDI and 122 with CSII.

Maternal metabolic control during pregnancy, gestational weight gain, insulin requirements, pregnancy outcome and neonatal status were compared between MDI and CSII arm.

The composite adverse neonatal outcome was diagnosed if at least one of the following was found: abnormal birth weight (LGA or SGA), congenital malformation, miscarriage, intrauterine fetal death, emergency CS due to fetal risk, iatrogenic prematurity, RDS, hypoglycemia, hyperbilirubinemia, and the postpartum $\mathrm{pH}$ in the umbilical artery $\leq 7.1$.

Results: The studied groups did not differ regarding gestational week at delivery, a proportion of births at full term, preterm births, miscarriages, or late pregnancy losses (intrauterine fetal death $>22$ weeks). Newborns of mothers treated with CSII showed lower incidence of neonatal complications (composite adverse neonatal outcome) compared to those of mothers treated with $\mathrm{MDI}(60 \% \mathrm{vs} 74 \%$, respectively; $\mathrm{p}=0.01)$. We did not find any association between the mode of treatment and composite adverse maternal outcome.

Conclusions: The use of CSII in the treatment of pregnant women with type 1 diabetes was associated with reduced number of neonatal complications presented as neonatal composite outcome but had no influence on maternal outcome. Key words: neonatal outcomes; pregestational diabetes mellitus; CSII; MDI
\end{abstract}

\section{INTRODUCTION}

Short after insulin introduction, perinatal mortality of newborns among mothers with type 1 diabetes (T1DM) reached $40 \%$. In the 1980 s, it dropped to $5 \%$ in the most advanced centers due to modern medical treatments, with a further reduction to $1-2 \%$ that is reported today [1]. Apart from this substantial improvement, pregnancy in women with type 1 diabetes is still associated with an elevated fetomaternal risk. Unfortunately, we are still far from pregnancy outcomes in patients with T1DM being comparable to the general population.

The number of miscarriages and birth defects in the diabetic population remains 2 to 4 times higher than in the healthy population [2-5]. Maternal hyperglycemia in later pregnancy is a risk factor for late intrauterine fetal death, which is 2 to 5 times higher than in the rest of the population. Moreover, small vessels disease, characteristic for long-lasting diabetes also induces changes in placental 
micro vessels, resulting in placental insufficiency that also contributes to an increased proportion of fetal demise [6]. Fetal macrosomia, occurring in $25-42 \%$ of newborns, is a consequence of increased glucose and lipid transfer and selective transport of amino acids through the placenta in the second half of pregnancy. Subsequent fetal hyperinsulinemia results in hypertrophy and hyperplasia of fetal cells [7]. Fetal hyperinsulinemia also inhibits the stimulating effects of cortisol and lowers lecithin levels, resulting in an impaired synthesis of surfactant and causing delayed fetal lung maturation. This impairment can manifest as respiratory distress syndrome (RDS) that occurs 4 to 6 times more often in that group of neonates and can also happen in deliveries at term. RDS coexists with other symptoms of diabetic fetopathy: hypoglycemia, polycythemia, and hyperbilirubinemia [8]. New data also link maternal diabetes to remote health risks in the offspring such as type 2 diabetes and obesity in later life, by altering intrauterine development and growth [9].

\section{Objectives}

An attempt was made to demonstrate the superiority of the treatment model using continuous subcutaneous insulin infusion (CSII) over multiple daily injections (MDI) of insulin in achieving a successful pregnancy outcome and good newborn's condition in patients with type 1 diabetes.

\section{MATERIAL AND METHODS}

A retrospective analysis based on medical records of the pregnancies and their outcome, and neonates born from 297 pregnant women with T1DM. Patients who developed type 1 diabetes at least one year before conception, with single pregnancy and were given obstetric care before the 15th week of gestation were included in the study. Participants were referred from local diabetic units for antenatal care with a diagnosis of type 1 diabetes set at a baseline by practitioners who initiated medical therapy. For perinatal risk assessment, we used modified White's classification of diabetes during pregnancy [10]. We compared the course of pregnancy, pregnancy outcome, and the newborn status in both groups, taking into account the efficacy of the two investigated treatment methods.

All patients were treated with functional intensive insulin therapy using recombinant human insulins or short-acting insulin analogues according to the uniform procedure of the study center.

We allocated our patients to two groups, according to the type of insulin therapy used:

1. The group of patients treated with multiple daily insulin injections; $\mathrm{N}=175$.

2. The group of patients treated with continuous subcutaneous insulin infusion; $\mathrm{N}=122$.
Each patient referred to the unit in early pregnancy between 2010 to 2015 for further antenatal care, was offered to continue MDI or to participate in appropriate training on CSII. In patients who completed the training, we commenced treatment with CSII. None of the participants used the continuous glucose monitoring system (CGMS).

According to the center's protocol, the patients were hospitalized at least once during each trimester and were also seen in the outpatient clinic for combined diabetes and obstetrics antenatal care, which enabled data collection throughout the entire pregnancy.

We used the following criteria for good diabetes control during pregnancy: glycated hemoglobin $\leq 6.1 \%$ (43 mmol/mol), fasting glucose $60-90 \mathrm{mg} / \mathrm{dL}(3.3-5.0 \mathrm{mmol} / \mathrm{L})$, glycemia one hour after a meal $<120 \mathrm{mg} / \mathrm{dL}(6.7 \mathrm{mmol} / \mathrm{L})$ and nocturnal glycemia $>60 \mathrm{mg} / \mathrm{dL}$ ( $3.3 \mathrm{mmol} / \mathrm{L}$ ), in accordance to the recommendations of the Polish Diabetes Association (PTD) of 2011 [11]. Patients kept self-monitoring diaries with commercially available glucometers determining capillary blood glucose levels. We diagnosed hypoglycemic events if glucose concentration was below $40 \mathrm{mg} / \mathrm{dL}$ ( $2.2 \mathrm{mmol} / \mathrm{L})$. Postprandial hyperglycemia was defined as a glycemia above $140 \mathrm{mg} / \mathrm{dL}$ (7.8 mmol/L) one hour after a meal.

We retrieved the following pieces of information from medical notes of the participants: patient age, age at diabetes onset, duration of illness, gestational age and body mass index (BMI) at the first antenatal visit, number of previous births, vascular complications like nephro- and retinopathy, and prepregnancy hypertension.

In subsequent trimesters, we monitored selected biochemical parameters evaluated at the Central Laboratory of the Hospital and daily glucose profiles performed by patients using glucometers for self-control during hospitalization.

From the medical records, we retrieved the following feto-maternal pregnancy outcomes: gestational age at delivery, mode of delivery, indications for caesarean sections, miscarriages and intrauterine deaths after 22nd gestational week. Women who lost their pregnancies within four weeks after enrollment were excluded from the analysis.

In newborns, we analyzed: birth weight, Apgar score at 1 and 5 minutes, pH from umbilical artery, birth weight (LGA; birthweight $>$ 90th percentile according to local growth charts for normal population, customized for gestational age at delivery and sex; SGA; birthweight < 10th percentile according to local growth charts for normal population, customized for gestational age at delivery and sex). We also recorded RDS, hyperbilirubinemia, hypoglycemia, and congenital malformations.

We defined the following abnormalities as malformations: cardiovascular defects (ventricular septal defect (VSD) 
and atrial septal defect (ASD), transposition of the great arteries (TGA), and coarctation of the aorta), caudal regression syndrome, and defects of the neural tube in the form of encephalocele [12]. We did not include newborns who have been diagnosed with patent foramen ovale because its prevalence was similar to this seen in the general population. Therefore, it could not be attributed to the maternal disease [13].

We defined a composite adverse neonatal outcome as abnormal birth weight (LGA or SGA), presence of congenital malformation, miscarriage, intrauterine fetal death, emergency CS due to fetal risk, iatrogenic prematurity, RDS, hypoglycemia, hyperbilirubinemia, and the postpartum $\mathrm{pH}$ in the umbilical artery $\leq 7.1$ [14].

The composite adverse maternal outcome was recorded if at least one of the following occurred: newly diagnosed gestational hypertension, preeclampsia, emergency cc due to maternal risk, a progression of vascular complications or diagnosis of vascular complications de novo, excessive gestational weight gain.

For the statistical analysis, we used tests appropriate for the distribution of the variables. Quantitative variables with normal distribution were presented as mean \pm SD or as median with minimum and maximum values for variables with nonparametric distribution. The chi ${ }^{2}$ test was used to compare the characteristics of the unrelated nominal variables. The Mann-Whitney test was used to compare the unrelated ordinal values. Logistic regression models were made to demonstrate the significance of the impact of the independent parameters on the occurrence of the studied end point. A relative risk analysis was performed to compare the two treatment methods. P values below 0.05 were considered significant. Statistical calculations were performed using Microsoft Excel 2010, Statistica 7.1, and SPSS 14.0.

\section{RESULTS}

Characteristics of participants did not show statistically significant differences in age, the onset of care, or baseline BMI between the groups. Pregnant women in the CSII arm were significantly younger when diagnosed with T1DM, had a significantly longer history of the disease and were significantly more likely to plan their pregnancies, comparing to the MDI arm. The CSII- group also included significantly more patients with long-lasting diabetes with vascular changes in comparison to the MDI group. (Tab. 1).

Daily insulin requirements and gestational weight gain were similar between the groups. We observed an improvement in metabolic control regardless of the type of therapy. A longitudinal analysis of $\mathrm{HbA} 1 \mathrm{c}$ showed a significant reduction in this parameter across the trimesters, irrespectively of the method of treatment. Also, mean daily glucose levels, fasting, postprandial and nocturnal glycemia improved in both arms. It is notable that fasting and nocturnal glycemia in the CSII group remained in the target range according to recommendations, but the results of MDI patients were higher [11]. We did not find differences in the number of hypoglycemic episodes during pregnancy among pregnant women from both groups. However, CSIl treatment was associated with a significantly lower incidence of postprandial hyperglycemia in the second (15\% vs $4.5 \%$ ) and in the third ( $22.5 \%$ vs $7.7 \%$ ) trimester compared to the MDI group (Tab. 2).

The studied groups did not differ regarding gestational week at delivery, a proportion of births at full term, preterm births, miscarriages, or late pregnancy losses. However, we noted a trend for the increased number of preterm births and intrauterine deaths in MDI arm (Tab. 3). In our cohort, we evaluated the composite adverse neonatal outcome between the groups and noted significantly reduced proportion of neonatal complications in the CSII group $(p<0.01)$ (Tab. 4). Maternal age, participant age when diagnosed with the disease and pregnancy planning (characteristics that differed significantly between the MDI and CSII groups, see Table 1) did not significantly correlate with composite neonatal outcome. Birth weight and Apgar scores were not statistically different, but we noted a trend for a higher first-minute Apgar score in CSII arm.

In the logistic regression model, we confirmed that duration of diabetes, the first-trimester body weight, glycated haemoglobin in the first and second trimesters, and insulin requirement in the last trimester were significant predictors

Table 1. Characteristics of the studied groups

\begin{tabular}{|c|c|c|c|}
\hline Analyzed parameter & $\begin{array}{l}\text { MDI } \\
N=175\end{array}$ & $\begin{array}{l}\mathrm{CSII} \\
\mathrm{N}=122\end{array}$ & $\mathbf{p}$ \\
\hline $\begin{array}{l}\text { Age of the pregnant } \\
\text { woman [years] }\end{array}$ & $28.0 \pm 4.9$ & $27.9 \pm 4.5$ & $0.96^{*}$ \\
\hline Disease onset [years] & $17.9 \pm 8.2$ & $14.5 \pm 6.9$ & $0.001^{*}$ \\
\hline Diabetes duration [years] & $10.2 \pm 6.8$ & $13.5 \pm 8.4$ & $0.0004^{*}$ \\
\hline Onset of diabetes care [w.g.] & $7.7 \pm 2.6$ & $7.4 \pm 2.4$ & $0.40^{*}$ \\
\hline $\begin{array}{l}\text { Body weight at the beginning } \\
\text { of care [kg] }\end{array}$ & $67.1 \pm 13.2$ & $65.8 \pm 11.3$ & $0.58^{*}$ \\
\hline $\begin{array}{l}\text { Weight gain during pregnancy } \\
{[\mathrm{kg}]}\end{array}$ & 12.58 & 12.06 & $0.50^{*}$ \\
\hline $\begin{array}{l}\text { BMl at the beginning of care } \\
{\left[\mathrm{kg} / \mathrm{m}^{2}\right]}\end{array}$ & $24.5 \pm 4.5$ & $23.8 \pm 3.4$ & $0.31^{*}$ \\
\hline $\begin{array}{l}\text { Patients planning pregnancy } \\
{[\mathrm{N} ; \%]}\end{array}$ & $55(30)$ & $55(45)$ & $0.02^{* *}$ \\
\hline $\begin{array}{l}\text { Patients with nephropathy } \\
{[\mathrm{N} ; \%]}\end{array}$ & $13(7)$ & $13(11)$ & $0.33^{* *}$ \\
\hline $\begin{array}{l}\text { Patients with diabetic } \\
\text { retinopathy }[\mathrm{N} ; \%]\end{array}$ & $25(14)$ & $20(16)$ & $0.62^{* *}$ \\
\hline $\begin{array}{l}\text { Patients with chronic } \\
\text { hypertension }[\mathrm{N} ; \%]\end{array}$ & $19(11)$ & $12(10)$ & $0.77^{* *}$ \\
\hline
\end{tabular}

* Mann-Whitney test, ${ }^{* *} \mathrm{Chi}^{2}$ test 


\begin{tabular}{|c|c|c|c|}
\hline Analyzed parameter & $\begin{array}{l}\text { MDI } \\
N=175\end{array}$ & $\begin{array}{l}\text { CSII } \\
N=122\end{array}$ & $\mathbf{p}$ \\
\hline \multicolumn{4}{|l|}{ Trimester I of pregnancy } \\
\hline $\begin{array}{l}\mathrm{HbA} 1 \mathrm{c}[\%] \\
\mathrm{HbA} 1 \mathrm{c}[\mathrm{mmol} / \mathrm{mol}]\end{array}$ & $\begin{array}{l}7.5 \pm 1.9 \\
58 \pm 20.9\end{array}$ & $\begin{array}{l}7.3 \pm 1.4 \\
56 \pm 15.4\end{array}$ & $0.61^{*}$ \\
\hline $\begin{array}{l}\text { Postprandial } \\
\text { hyperglycemia } \geq 140 \mathrm{mg} / \mathrm{dL} \\
{[\mathrm{N} ; \%]}\end{array}$ & $110(27)^{* * *}$ & $86(27.5)^{* * *}$ & $0.68^{* *}$ \\
\hline \multicolumn{4}{|l|}{ Trimester II of pregnancy } \\
\hline $\begin{array}{l}\mathrm{HbA} 1 \mathrm{c}[\%] \\
\mathrm{HbA} 1 \mathrm{c}[\mathrm{mmol} / \mathrm{mol}]\end{array}$ & $\begin{array}{l}6.1 \pm 1.0 \\
43 \pm 11\end{array}$ & $\begin{array}{l}6.0 \pm 0.9 \\
42 \pm 10\end{array}$ & $0.97^{*}$ \\
\hline $\begin{array}{l}\text { Postprandial } \\
\text { hyperglycemia } \geq 140 \mathrm{mg} / \mathrm{dL} \\
{[\mathrm{N} ; \%]}\end{array}$ & $53(15)^{* * *}$ & $14(4.5)^{* * * *}$ & $0.0001^{* *}$ \\
\hline \multicolumn{4}{|l|}{ Trimester II of pregnancy } \\
\hline $\begin{array}{l}\mathrm{HbA} 1 \mathrm{c}[\%] \\
\mathrm{HbA} 1 \mathrm{c}[\mathrm{mmol} / \mathrm{mol}]\end{array}$ & $\begin{array}{l}6.5 \pm 1.1 \\
48 \pm 12\end{array}$ & $\begin{array}{l}6.3 \pm 0.7 \\
45 \pm 8\end{array}$ & $0.67^{*}$ \\
\hline $\begin{array}{l}\text { Postprandial } \\
\text { hyperglycemia } \geq 140[\mathrm{mg} / \mathrm{dL}]\end{array}$ & $64(22.5)^{* * *}$ & $21(7.7)^{* * *}$ & $0.0001^{* *}$ \\
\hline \multicolumn{4}{|c|}{$\begin{array}{l}{ }^{*} \text { Mann-Whitney test, }{ }^{* *} \mathrm{Chi}^{2} \text { test, }{ }^{* * *} \% \text { calculated for the number of all } \\
\text { measurements from a given group in a given trimester. } \\
\text { In the second trimester, calculations were made for MDI N }=165, \mathrm{CSII} \\
\mathrm{N}=120 \text { (miscarriage excluded) and in the third trimester, calculations were } \\
\text { made for MDI N }=161, \mathrm{CSII} \mathrm{N}=119 \text { (intrauterine death excluded) }\end{array}$} \\
\hline
\end{tabular}

\begin{tabular}{|c|c|c|c|}
\hline Analyzed parameter & $\begin{array}{l}\text { MDI } \\
N=175\end{array}$ & $\begin{array}{l}\text { CSII } \\
N=122\end{array}$ & $\mathbf{p}$ \\
\hline Gestational age at delivery [w.g.] & $37.5 \pm 2.0$ & $37.5 \pm 1.9$ & $0.63^{*}$ \\
\hline Births at full term [N; \%] & $131(73)$ & 95 (77.5) & $0.64^{*}$ \\
\hline Late preterm births: GA 34-37 [N; \%] & $17(9)$ & $19(15.5)$ & $0.18^{*}$ \\
\hline Preterm births: $\mathrm{GA} \leq 33+6[\mathrm{~N} ; \%]$ & $13(7)$ & $5(4)$ & $0.35^{*}$ \\
\hline Miscarriages: GA < 22 [N; \%] & $10(9)$ & $2(2)$ & $0.15^{*}$ \\
\hline $\begin{array}{l}\text { Intrauterine deaths below } 22 \text { nd } \\
\text { gestational week }[\mathrm{N} ; \%]\end{array}$ & $4(2)$ & $1(1)$ & $0.90^{*}$ \\
\hline
\end{tabular}

${ }^{*} \mathrm{Chi}^{2}$ test

of excessive fetal birth weight in the studied population (Tab. 5). In a separate analysis the proportion of LGA newborns was similar between the arms.

Late pregnancy loss (intrauterine death) occurred in five patients in the studied population. Four cases recorded in the MDI arm occurred in patients with an inadequate metabolic control throughout the whole pregnancy (mean HbA1c in a subgroup of 9.7\%) or noncomplying (not reporting for scheduled antenatal check-ups in the referral center). A single case noted in the CSII arm occurred in a well-controlled woman with long-lasting diabetes and coexisting diabetic kidney disease which is an independent risk factor for unfavorable neonatal outcome.

We did not note any association between the mode of treatment end composite adverse maternal outcome.

\begin{tabular}{|l|l|l|l|}
\hline \multicolumn{3}{|l|}{ Table 4. Status of newborns } & \multicolumn{3}{l|}{ MDI } \\
\hline Analyzed parameter & N $=161$ & $\begin{array}{l}\text { CSII } \\
\text { N }=119\end{array}$ & p \\
\hline Birth weight [g] & $3480 \pm 750$ & $3430 \pm 680$ & $0.58^{*}$ \\
\hline $\begin{array}{l}\text { Apgar score in the first min. of } \\
\text { life [median, min-max] }\end{array}$ & $8[1-10]$ & $8[1-10]$ & $0.06^{*}$ \\
\hline $\begin{array}{l}\text { Apgar score in the fifth min. of } \\
\text { life [median, min-max] }\end{array}$ & $9[1-10]$ & $9[1-10]$ & $0.38^{*}$ \\
\hline $\begin{array}{l}\text { Composite adverse neonatal } \\
\text { outcome [N; \%] }\end{array}$ & $119(74)$ & $72(60)$ & $0.01^{* *}$ \\
\hline
\end{tabular}

Miscarriages and intrauterine death excluded from the analysis Composite adverse neonatal outcome: abnormal birth weight (LGA or SGA) malformation, miscarriage, intrauterine fetal death, emergency CS due to fetal risk, iatrogenic prematurity, RDS, hypoglycemia, hyperbilirubinemia, and the postpartum $\mathrm{pH}$ in the umbilical artery $\leq 7.1$.

* Mann-Whitney test, ${ }^{* *}$ Chi ${ }^{2}$ test

\begin{tabular}{|l|l|l|l|}
\hline Table 5. Predictors of LGA & OR & $\mathbf{( 9 5 \% ~ O R ) ~}$ & p \\
\hline Analyzed parameter & 0.95 & $(0.92-0.99)$ & 0.02 \\
\hline Diabetes duration [years] & 0.22 & $(0.08-0.57)$ & 0.00 \\
\hline Body weight in trimester I [kg] & 1.99 & $(1.09-3.62)$ & 0.00 \\
\hline HbA1c in trimester I [\%] & 0.29 & $(0.18-0.48)$ & 0.01 \\
\hline HbA1c in trimester II [\%] & 2.63 & $(1.05-6.63)$ & 0.04 \\
\hline $\begin{array}{l}\text { Insulin requirement in trimester III } \\
\text { (kg/body weight) }\end{array}$ & & & \\
\hline
\end{tabular}

\section{DISCUSSION}

Hormonal changes, mainly increasing concentrations of anti-insulin factors, can lead to significant fluctuations in glucose levels, even in patients whose metabolism is considered well-controlled before pregnancy. Moreover, during normal pregnancy maternal body operates on lower glucose levels due to natural hormonal adaptations that give a fetus a priority in an access to maternal glucose [15]. Therefore, close monitoring of both maternal and fetal well-being is mandatory, as there is a higher risk of miscarriage, congenital malformations, early and late intrauterine death, and fetal growth disorders that can occur even at nearly normal glycemic levels [10, 11, 16].

The current standards concerning antenatal care for women with diabetes recommend the use of intensive functional insulin therapy in this population. Taking into account the patient's clinical condition, technical ability, and compliance, we have two options for administering insulin: using MDI or CSII.

Carbohydrate metabolism changes in the pregnant woman and the pregnancy itself becomes a diabetogenic factor, altering the hormonal homeostasis. There is a worsening of carbohydrate tolerance, increased peripheral insulin resistance, and thus impaired action of exogenous insulin $[17,18]$. Pregnant women must be aware, however, that continuous adding extra insulin doses to achieve normo- 
glycemia is a vicious cycle that raises insulin resistance. This wrong habit also leads to an excessive gestational body weight gain and adds to dangerous changes in lipid profile. This way, inappropriate insulin dose also contributes to metabolic changes that often lead to excessive fetal growth, despite apparently good metabolic control [19].

An important problem during pregnancy complicated by pregestational diabetes is the abnormal growth of the fetus which can manifest as excessive (LGA) or restricted (SGA) growth. Excessive fetal growth is caused mainly by fetal hyperinsulinemia, resulting from maternal hyperglycemia [20]. However, studies on metabolic control during pregnancy as a predictor of growth disorders provide contradictory results, whether it is more influenced by the average glycemia or pre- or postprandial glycemia. Some authors confirm that elevated $\mathrm{HbA} 1 \mathrm{c}$ levels are a significant risk indicator of LGA [21, 22]. Combs et al. [23] deny, however, the importance of average glycemia, and point out especially on postprandial hyperglycemia. In recent reports, LGA newborns are predominant in pregnancies complicated by mild hyperglycemia and maternal obesity, whereas the increased prevalence of SGA is characteristic for pregnancies complicated by diabetic microangiopathy, especially diabetic kidney disease, as well as hypertension and preeclampsia $[24,25]$. These observations demonstrate the contribution of factors other than glycemia to fetal growth abnormalities, i.e. obesity and accompanying lipid disorders and the role of vascular complications. Also, in our cohort, we did not find any significant differences between the mean birth weight in the MDI-group and those of the CSII group Our results are consistent with the results of Cypryk et al. [26], who did not show the effect of type of insulin therapy on an occurrence of either LGA and SGA in their group. It should be noted, however, that in the CSII, the proportion of LGA was a few percent lower than in the MDI group, while the percentage of SGA was very similar in both groups although the CSII group consisted of patients with more complicated diabetes. The lack of significant differences in the incidence of growth disorders does not, however, make it possible to unequivocally demonstrate the superiority of any of the analyzed methods of intensive functional insulin therapy. However, due to the fact that the percentage of all growth disorders was lower in the CSII group, it seems that this model of treatment may contribute to a reduction in the incidence of these disorders.

In the logistic regression model, the predictors of LGA were: duration of diabetes, patient body weight in the first trimester, glycated haemoglobin in the first and second trimesters, and insulin requirement in the last trimester of pregnancy. These associations provide an additional proof that in an appropriately controlled pregnant woman, the occurrence of excessive fetal birth weight depends on many factors and the fact that the insulin requirement in the third trimester of pregnancy was the most potent predictor confirms the role of insulin resistance in inducing excessive growth. Low body weight in pregnant women seems to correlate with the fact that these patients often pay less attention to later weight gain in pregnancy than overweight patients, and we know that excessive weight gain is also a factor inducing excessive fetal growth.

Assessment of pregnancy outcomes including newborn birth weight, week of delivery, and the mode of delivery, did not show statistically significant differences between the MDI and CSII groups, which is in agreement with the results of other authors [18, 27-29]. The timing and mode of delivery in patients with T1DM remains a subject to extensive discussion. In the absence of indications resulting from vascular complications, fetal macrosomia, or birth defects, there are no indications for scheduled cesarean section [11]. The situation can change completely intrapartum when symptoms of fetal or maternal risks can occur suddenly. Neff et al. showed a significant difference in the higher proportion of pregnancies completed by cesarean section in CSII patients. However, this was a group of patients with a significantly longer disease history and was, therefore, more burdened by vascular complications [30]. In our cohort, we did not find any significant difference in the proportion of physiological labours, labours with interventions, or cesarean section.

Most of the available reports show no effect of the used therapy on the newborn status after birth [18, 26, 30, 31]. Tylaviya et al. [32] demonstrated that the only significant parameter is the Apgar score, which was higher in CSII mothers. Several reasons make any epidemiological analysis of pregnancy outcomes in diabetic mothers challenging: first, CSII is a relatively new modality in diabetes treatment, and only limited amount of data from small cohorts of pregnant women is available. Second, due to a general improvement in antenatal care seen in the last decades in the settings where specific needs of pregnant women are appropriately addressed, some of the perinatal complications (e,g. late intrauterine death or intrapartum complications) became rare anyway. Therefore, prospective intervention trials adequately powered to track rare complications in a population of pregnant women suffering from the disease complicating ca. $1 \%$ of pregnancies would be trying even if running such research in a frame of multicenter cooperation. In our cohort, we found a significantly reduced number of overall neonatal complications, defined as a composite adverse neonatal outcome, in the CSII arm. Although this difference lost statistical significance after controlling for the confounded, it still remains a possible relevant clinical finding. The reasons for this advantage should be sought in facilitated glycemic control with the use of insulin pump that accurately adjusts 
the dose of both the basal insulin and the type and dose of prandial boluses [33]. Continuous infusion allows for a more flexible and stable administration of basal insulin achieving nocturnal and early-morning normoglycemia more efficiently than long-acting insulin boluses administered with a pen at night. Also, reduced number of hyperglycemia noted in our CSII arm could suggest improved glycemic variability, i.e. diminished short-term glucose fluctuations that can translate into a better endothelial function in placenta and more stable placental transfer of nutrients. All this contributes to the optimization of treatment and the achievement of better metabolic control that alleviates or substantially reduces potent adverse effects of hyperglycemia on the fetus $[18,27,34]$.

\section{CONCLUSIONS}

Treatment of type 1 diabetic pregnant women with a personal insulin pump allows for the optimization of therapy and precise titration of the basal insulin dose and the type and size of the prandial boluses.

As a result, we note a decreased incidence of hyperglycemia, which seems to reduce the risk of composite adverse neonatal outcome and early postpartum complications in the offspring in this group of patients.

\section{REFERENCES}

1. Feig DS, Palda VA. Type 2 diabetes in pregnancy: a growing concern. Lancet. 2002; 359(9318): 1690-1692, doi: 10.1016/S0140-6736(02)085999, indexed in Pubmed: 12020549.

2. Wender-Ożegowska E. Maternal diseases complicating the course of pregnancy, Ed. Bręborowicz G. In: Diabetes. In: Obstetrics. Volume 2. Maternal-fetal medicine. . PZWL Medical Publishing, Warsaw 2012: 425-451.

3. Dunne F. Pregestational Diabetes Mellitus and Pregnancy. Trends in Endocrinology \& Metabolism. 1999; 10(5): 179-182, doi: 10.1016/s10432760(98)00147-7.

4. Suhonen L, Hiilesmaa V, Teramo K. Glycaemic control during early pregnancy and fetal malformations in women with type I diabetes mellitus. Diabetologia. 2000;43(1): 79-82, doi: 10.1007/s001250050010, indexed in Pubmed: 10663219.

5. Miodovnik M, Lavin JP, Knowles HC, et al. Spontaneous abortion among insulin-dependent diabetic women. Am J Obstet Gynecol. 1984; 150(4): 372-376, indexed in Pubmed: 6207729.

6. Confidential Enquiry into Maternal and Child Health: Pregnancy in Women with Type 1 and Type 2 Diabetes in 2002-2003 in England, Wales and Northern Ireland. CEMACH London 2005. https://stratog.rcog.org. uk/sites/default/files/Diabetes\%20and\%20other\%20endocrinopathies/CEMACH_Pregnancy_type_1_2_diabetes.pdf.

7. Czeszyńska MB. Newborn of a mother with diabetes - past, present, future. Pediatric Clinic. 2006; 76: 828-837.

8. Manderson JG, Mullan B, Patterson CC, et al. Cardiovascular and metabolic abnormalities in the offspring of diabetic pregnancy. Diabetologia. 2002; 45(7): 991-996, doi: 10.1007/s00125-002-0865-y, indexed in Pubmed: 12136397.

9. Wroblewska-Seniuk K, Wender-Ozegowska E, Szczapa J. Long-term effects of diabetes during pregnancy on the offspring. Pediatr Diabetes. 2009; 10(7): 432-440, doi: 10.1111/j.1399-5448.2009.00507.x, indexed in Pubmed: 19476566.

10. White P. Pregnancy complicating diabetes. The American Journal of Medicine. 1949; 7(5): 609-616, doi: 10.1016/0002-9343(49)90382-4.

11. Clinical recommendations for management of diabetic patients, 2011. Practical Diabetology. 2011; 12(A): 1-46.
12. Wilczyński J, Cypryk K, Cyranowicz B. Incidence of birth defects in neonates of mothers with insulin-dependent diabetes. In: Słomko Z. ed. Materials of the I Congress of the Polish Society of Perinatal Medicine. PAN, Poznań 1995: 175-178.

13. Meissner I, Whisnant JP, Khandheria BK, et al. Prevalence of potential risk factors for stroke assessed by transesophageal echocardiography and carotid ultrasonography: the SPARC study. Stroke Prevention: Assessment of Risk in a Community. Mayo Clin Proc. 1999; 74(9): 862-869, indexed in Pubmed: 10488786.

14. Ferreira-González I, Permanyer-Miralda G, Busse JW, et al. Methodologic discussions for using and interpreting composite endpoints are limited, but still identify major concerns. J Clin Epidemiol. 2007; 60(7): 651-7; discussion 658, doi: 10.1016/j.jclinepi.2006.10.020, indexed in Pubmed: 17573977.

15. Yogev Y, Ben-Haroush A, Chen R, et al. Diurnal glycemic profile in obese and normal weight nondiabetic pregnant women. Am J Obstet Gynecol. 2004; 191(3): 949-953, doi: 10.1016/j.ajog.2004.06.059, indexed in Pubmed: 15467570.

16. Colstrup $M$, Mathiesen ER, Damm P, et al. Pregnancy in women with type 1 diabetes: have the goals of St. Vincent declaration been met concerning foetal and neonatal complications? J Matern Fetal Neonatal Med. 2013; 26(17): 1682-1686, doi: 10.3109/14767058.2013.794214, indexed in Pubmed: 23570252.

17. Wender-Ożegowska E. Factors affecting fetal development during pregnancy complicated with diabetes mellitus. Supplement: Clinical Perinatology and Gynecology. ed. Słomko Z. The Scientific Publishers, Poznań 2000.

18. Wender-Ozegowska E, Zawiejska A, Ozegowska K, et al. Multiple daily injections of insulin versus continuous subcutaneous insulin infusion for pregnant women with type 1 diabetes. Aust N Z J Obstet Gynaecol. 2013; 53(2): 130-135, doi: 10.1111/ajo.12027, indexed in Pubmed: 23316799.

19. Saisho Y, Tanaka C, Tanaka K, et al. Relationships among different glycemic variability indices obtained by continuous glucose monitoring Prim Care Diabetes. 2015; 9(4): 290-296, doi: 10.1016/j.pcd.2014.10.001, indexed in Pubmed: 25456706.

20. Lepercq J, Taupin P, Dubois-Laforgue D, et al. Heterogeneity of fetal growth in type 1 diabetic pregnancy. Diabetes Metab. 2011; 27(3): 339-344, indexed in Pubmed: 11431599.

21. Johnstone FD, Mao JH, Steel JM, et al. Factors affecting fetal weight distribution in women with type I diabetes. BJOG. 2000; 107(8): 1001-1006, indexed in Pubmed: 10955432.

22. Small M, Cameron A, Lunan CB, et al. Macrosomia in pregnancy complicated by insulin-dependent diabetes mellitus. Diabetes Care. 1987; 10(5): 594-599, indexed in Pubmed: 3677977.

23. Combs CA, Gunderson E, Kitzmiller JL, et al. Relationship of fetal macrosomia to maternal postprandial glucose control during pregnancy. Diabetes Care. 1992; 15(10): 1251-1257, indexed in Pubmed: 1425084.

24. Martínez-Abundis E, González-Ortiz M, Pascoe-González S. Serum leptin levels and the severity of preeclampsia. Arch Gynecol Obstet. 2000; 264(2): 71-73, indexed in Pubmed: 11045326.

25. Gonzalez-Gonzalez NL, Ramirez O, Mozas J, et al. Factors influencing pregnancy outcome in women with type 2 versus type 1 diabetes mellitus. Acta Obstet Gynecol Scand. 2008; 87(1): 43-49, doi: 10.1080/00016340701778732, indexed in Pubmed: 18158626.

26. Cypryk K, Kosiński M, Kamińska P, et al. Diabetes control and pregnancy outcomes in women with type 1 diabetes treated during pregnancy with continuous subcutaneous insulin infusion or multiple daily insulin injections. Polish Archives of Internal Medicine. 2008; 118(6): 229-344, doi: 10.20452/pamw.408.

27. Evers IM, de Valk HW, Mol BWJ, et al. Macrosomia despite good glycaemic control in Type I diabetic pregnancy; results of a nationwide study in The Netherlands. Diabetologia. 2002; 45(11): 1484-1489, doi: 10.1007/s00125-002-0958-7, indexed in Pubmed: 12436330.

28. Kernaghan D, Farrell T, Hammond $\mathrm{P}$, et al. Fetal growth in women managed with insulin pump therapy compared to conventional insulin. Eur J Obstet Gynecol Reprod Biol. 2008; 137(1): 47-49, doi: 10.1016/j. ejogrb.2007.05.006, indexed in Pubmed: 18006209.

29. Giménez M, Conget I, Nicolau J, et al. Outcome of pregnancy in women with type 1 diabetes intensively treated with continuous subcutaneous insulin infusion or conventional therapy. A case-control study. Acta Diabetol. 2007; 44(1): 34-37, doi: 10.1007/s00592-007-0239-5, indexed in Pubmed: 17357884

30. Neff KJ, Forde R, Gavin C, et al. Pre-pregnancy care and pregnancy outcomes in type 1 diabetes mellitus: a comparison of continuous 
subcutaneous insulin infusion and multiple daily injection therapy. Ir J Med Sci. 2014; 183(3): 397-403, doi: 10.1007/s11845-013-1027-6, indexed in Pubmed: 24142539.

31. Bruttomesso D, Bonomo M, Costa S, et al. Italian Group for Continuous Subcutaneous Insulin Infusion in Pregnancy. Type 1 diabetes control and pregnancy outcomes in women treated with continuous subcutaneous insulin infusion (CSII) or with insulin glargine and multiple daily injections of rapid-acting insulin analogues (glargine-MDI). Diabetes Metab. 2011;37(5): 426-431, doi: 10.1016/j.diabet.2011.02.002, indexed in Pubmed: 21474360.

32. Talaviya PA, Saboo BD, Joshi SR, et al. Pregnancy outcome and glycemic control in women with type 1 diabetes: a retrospective comparison between CSII and MDI treatment. Diabetes Metab Syndr. 2013; 7(2): 68-71, doi: 10.1016/j.dsx.2013.02.032, indexed in Pubmed: 23680243.

33. Kekäläinen $P$, Juuti $M$, Walle $T$, et al. Continuous Subcutaneous Insulin Infusion During Pregnancy in Women with Complicated Type 1 Diabetes Is Associated with Better Glycemic Control but Not with Improvement in Pregnancy Outcomes. Diabetes Technol Ther. 2016; 18(3): 144-150, doi: 10.1089/dia.2015.0165, indexed in Pubmed: 26502287.

34. Lapolla A, Dalfrà MG, Masin M, et al. Analysis of outcome of pregnancy in type 1 diabetics treated with insulin pump or conventional insulin therapy. Acta Diabetol. 2003; 40(3): 143-149, doi: 10.1007/s00592-0030103-1, indexed in Pubmed: 14605971. 